\title{
Comparison of the Effectiveness of Three Methods of Recanalization in a Model of the Middle Cerebral Artery: Thrombus Aspiration via a 4F Catheter, Thrombus Aspiration via the GP Thromboaspiration Device, and Mechanical Thrombectomy Using the Solitaire Thrombectomy Device
}

\author{
Christopher Tennuci, ${ }^{1,2}$ Gillian Pearce, ${ }^{3}$ Julian Wong, ${ }^{4,5}$ Sanjeev Nayak,, 6 \\ Tom Jones, ${ }^{1,5}$ Frank Lally, ${ }^{2}$ and Christine Roffe ${ }^{2,5}$ \\ ${ }^{1}$ School of Medicine, Keele University, Staffordshire ST5 5BG, UK \\ ${ }^{2}$ Institute for Science and Technology in Medicine, Keele University, Keele, Staffordshire ST6 6TP, UK \\ ${ }^{3}$ Biomedical Science Division, University of Wolverhampton, Wolverhampton WV1 1LY, UK \\ ${ }^{4}$ Department of Vascular Surgery, University Hospital of North Staffordshire, Newcastle Road, Staffordshire ST4 6QG, UK \\ ${ }^{5}$ Stroke Research Offices, North Staffordshire Royal Infirmary, Princes Road, Stoke-on-Trent, Staffordshire ST4 7LN, UK \\ ${ }^{6}$ Neuroradiology, University Hospital North Staffordshire, Princes Road, Stoke-on-Trent, Staffordshire ST4 7LN, UK \\ Correspondence should be addressed to Christine Roffe, christine.roffe@northstaffs.nhs.uk
}

Received 29 November 2010; Revised 25 January 2011; Accepted 17 February 2011

Academic Editor: Graham S. Venables

Copyright ( $\odot 2011$ Christopher Tennuci et al. This is an open access article distributed under the Creative Commons Attribution License, which permits unrestricted use, distribution, and reproduction in any medium, provided the original work is properly cited.

Introduction. This paper compares different approaches to recanalization in a model of the middle cerebral artery (MCA). Methods. An occlusive thrombus (lamb's blood) was introduced into the MCA of a model of the cerebral circulation perfused with Hartmann's solution ( 80 pulsations/min, mean pressure $90 \mathrm{~mm} \mathrm{Hg}$ ). Three methods of clot retrieval were tested: thrombus aspiration via a $4 \mathrm{~F}$ catheter $(n=26)$, thrombus aspiration via the GP thrombus aspiration device $(\mathrm{GPTAD})(n=30)$, and mechanical thrombectomy via the Solitaire Device $(n=30)$. Results. Recanalization rate was similar for all 3 approaches $(62 \%$, $77 \%$, and $85 \%$ ). Time to recanalization was faster with aspiration devices (41 SD $42 \mathrm{~s}$ for 4F and 61 SD $21 \mathrm{~s}$ for GPTAD) than with the Solitaire (197 SD 64 s $P<.05$ Kruksal-Wallis). Clot fragmentation was the same in the Solitaire (23\%) and the GPTAD (23\%), but higher with the $4 \mathrm{~F}(53 \%, P<.05)$. Conclusion. In this model, thrombus aspiration was faster than mechanical thrombectomy, and similarly effective at recanalization. These results should be confirmed in vivo.

\section{Introduction}

The aim of treatment of an acute ischaemic stroke is to recanalize the occluded cerebral artery to restore cerebral perfusion. This limits the damage to the cerebral tissue, reduces infarct size, and therefore improves long-term outcome [1]. There is no sparing of cerebral tissue damage beyond 6-7 hours or longer after onset [2].

Intravenous thrombolysis using recombinant tissue plasminogen activator (rt-PA) within 4.5 hours of onset of symptoms of acute stroke has been shown to improve out- come significantly [3]. Recanalization rates vary from 30 to $92 \%$ during the first 6-24 hours after treatment with intravenous thrombolysis [1]. However, there are drawbacks to using thrombolysis, such as the short time window for treatment and the risk of intracerebral (ICH) and systemic haemorrhage. This has limited the number of stroke patients receiving thrombolysis to an estimated $4 \%$ in Europe [3].

Thrombectomy involves the recanalization of an artery via endovascular extraction of the thrombus through a catheter using a mechanical thrombectomy device (MTD) [4]. This is an alternative approach to clot removal and may 


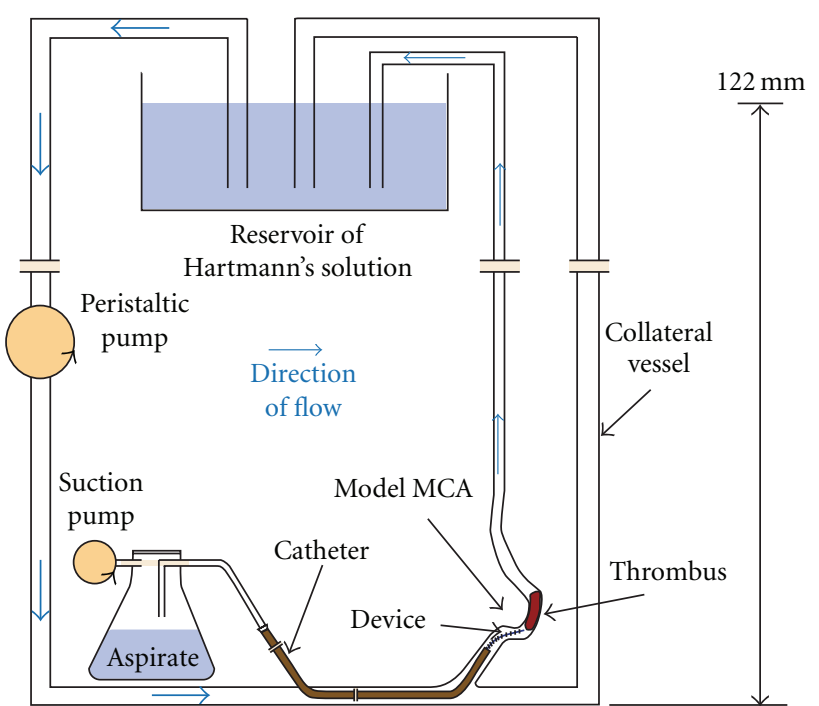

FIGURE 1: Diagram of the pulsatile flow system. Blue arrows indicate direction of perfusate flow.

overcome some of the problems above [5]. Thrombectomy is considered if patients present beyond the approved treatment window for intravenous thrombolysis, if they fail to recanalize after IV thrombolysis, or if they have contraindications to thrombolysis. The time limit for thrombectomy after middle cerebral artery (MCA) occlusion is 6-8 hours after symptom onset [1], thus providing a significantly longer intervention opportunity.

Problems with thrombectomy include the need for sedation/anaesthesia, the time taken to organize and perform the procedure, difficulties in navigation of the device intracranially, and in capture of the thrombi within the tortuous carotid arteries and intracranial vessels. Furthermore, devices may fracture leaving it difficult to retrieve components and there is a risk of damage to the artery wall, causing haemorrhage or vasospasm and a risk of distal embolization due to clot fragmentation, causing further ischaemia $[2,6,7]$.

The GP thrombus aspiration device (GPTAD) [8] may overcome some of the problems associated with mechanical thrombectomy. It has no moving parts, thus reducing the risk of device fracture. It does not enter or disrupt the clot and allows aspiration during clot retrieval, reducing the risk of loose fragments. The vortex pattern flow generated by the GPTAD during suction is associated with low forces at the periphery of the device reducing the risk of arterial collapse [9-11]. An earlier study [12] demonstrated (i) that the GPTAD (previously called the GP MTD) can be effectively maneuvered though a model of the larger intracerebral arteries, (ii) that there is a correlation between the pressure required for extraction and the mass of the thrombus, (iii) that thrombus removal via the GPTAD can be further enhanced by the addition of alteplase to the perfusate, and (iv) that the use of the GPTAD does not damage the arterial wall of abattoir porcine artery walls studied in-vitro with suction pressures of up to $30 \mathrm{KPa}$. These experiments have

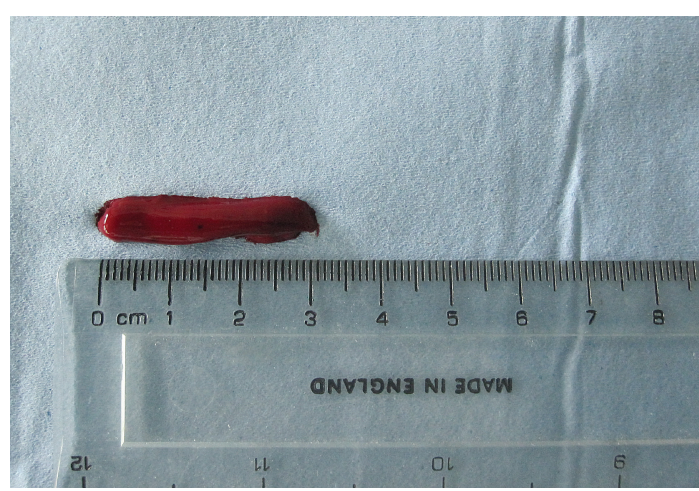

Figure 2: Prepared clot.

shown that thrombus aspiration via the GPTAD is feasible and more effective than aspiration through a simple catheter in vitro.

The aim of this study is to compare the effectiveness of thrombus aspiration using the GPTAD and a simple catheter with mechanical thrombectomy using the Solitaire Device, a retrievable, detachable stent-platform-based thrombectomy device already in clinical use within Europe [13, 14].

\section{Methods}

Three methods of recanalization of an occluded vessel were tested in a pulsatile model of the cerebral circulation.

2.1. The Pulsatile Flow System. The experimental pulsatile flow system used to simulate the cerebral circulation (Figure 1) has been previously described [15]. It was perfused with Hartmann's solution (Baxter Healthcare Ltd., Thetford, Norfolk, UK) and placed in a beaker in a water bath (Grant Instruments Cambridge, UK) at $37^{\circ} \mathrm{C}$. A peristaltic pump (Watson Marlow, Falmouth, Cornwall, UK) was used to create a pulsatile flow of 80 pulsations per minute. A silicone model of the middle cerebral artery (MCA) with an internal diameter of $3 \mathrm{~mm}$ (ELSTRAT Geneva, Switzerland) was integrated into the flow system. The perfusion pressure was set to match a mean arterial pressure (MAP) of $90 \mathrm{~mm} \mathrm{Hg}$ using a hydrostatic pressure adjustment. To do this, a second circulation in parallel to the existing circulation with the MCA was set up with an outflow $122 \mathrm{~cm}(122 \mathrm{~cm}$ $\mathrm{H}_{2} \mathrm{O} / 1.36 \mathrm{~mm} \mathrm{Hg}=90 \mathrm{~mm} \mathrm{Hg}$ ) above the MCA.

\subsection{Thrombus Preparation and Loading into the Pulsatile Flow} System. Lambs' blood (AJ Greens Abattoir, Stoke, UK) was allowed to coagulate for $24 \mathrm{~h}$, shaped using a scalpel into clots of an approximately $5 \mathrm{~mm}$ diameter and $30 \mathrm{~mm}$ length (Figure 2), washed in Hartmann's solution, and drip dried. Clot weight was recorded using electronic scales (Ohaus, Pinebrook, USA) before and after introduction into the experimental vascular system.

The clot was placed in the reservoir of Hartmann's solution, with the peristaltic pump turned off. The pump was then turned on with flow in the reverse direction to 


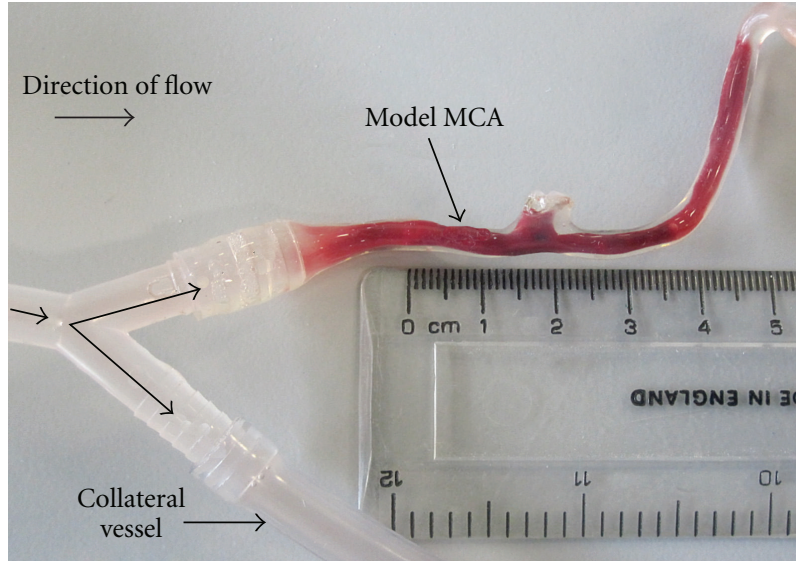

Figure 3: MCA with thrombus loaded and collateral vessel displayed.

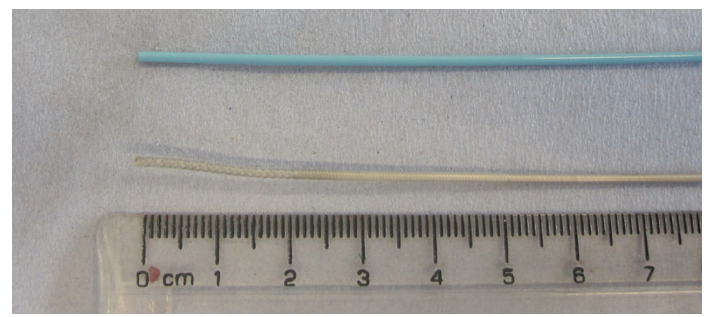

FIGURE 4: Devices used for clot aspiration: the 4F catheter (top) and the GPTAD (below).

physiological flow allowing the thrombus to travel along the collateral vessel past the model MCA. The pump was then turned off, and the collateral vessel was occluded using a clamp. The pump was turned back on with flow in the correct physiological direction causing the thrombus to enter and occlude the model MCA. The final thrombus length was then measured in situ using a ruler (Figure 3). The peristaltic pump was turned on $(80 \mathrm{bpm})$, and the collateral vessel opened to resume flow [15].

2.3. Devices and Techniques Used for Recanalization. Devices used for thrombus aspiration are shown in Figure 4. The Gwen Pearce thrombus aspiration device (GPTAD) (Pearce G, Perkinson N, inventors; US patent application 0509583.1.2005) is an embedded thrombectomy device of $1 \mathrm{~mm}$ diameter within a $4 \mathrm{~F}$ catheter, with a helical engraving on its internal surface (KIMAL, Uxbridge, UK). The GPTAD was introduced into the silicone tubing leading to the model MCA through a shortened $(20 \mathrm{~cm}) 6 \mathrm{~F}$ catheter, which was used as a sheath $(10 \mathrm{~cm}$ inside and $10 \mathrm{~cm}$ outside the entry point into the model vascular system) and advanced towards the clot. When the catheter tip was within $3-5 \mathrm{~mm}$ of the proximal aspect of the clot, the suction pump, set at $50 \mathrm{kPa}$, was turned on. The thrombus was then aspirated into the device tip and removed from the model MCA attached to the catheter tip. This was done in a controlled way under constant traction to avoid loss of contact with the clot during

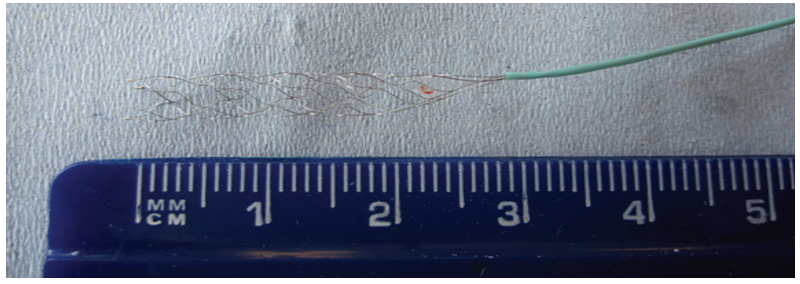

(a)

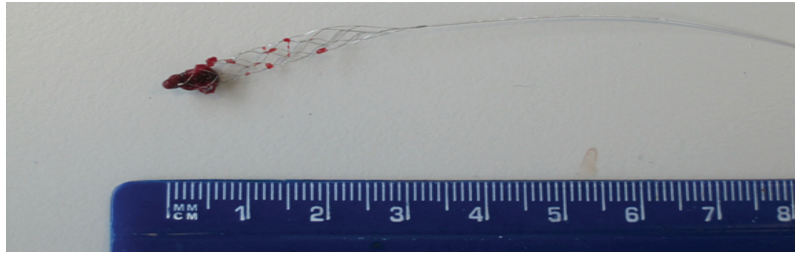

(b)

FIgure 5: Solitaire AB Device expanded (a) and after clot retrieval (b).

extraction. After removal of the $4 \mathrm{~F}$ catheter, the sheath stayed in place for subsequent experiments. A $4 \mathrm{~F}$ catheter $(4 \mathrm{~F})$ attached to a suction pump was used as a control. This was inserted through the $6 \mathrm{~F}$ sheath, advanced towards the clot, and withdrawn in the same way as described for the GPTAD. The same suction pressure of $50 \mathrm{kPa}$ was applied to aspirate the clot.

Mechanical thrombectomy was performed using the Solitaire AB Device (Figure 5(a)), a self expanding retrievable stent (ev3 Inc., Irvine, California), using techniques shown in the Solitaire operating guideline [16]. An 8F catheter, connected to a rheostatic valve, was introduced into the silicone tube leading to the model MCA. Through this, a 0.014-inch guide wire (Boston Scientific, FA, USA) was threaded and advanced to pass between the vessel wall and thrombus, to a final position just beyond the thrombus. A microcatheter $\left(0.021^{\prime \prime}\right)$ connected to a rheostatic valve was guided beyond the thrombus using the microguidewire. The Solitaire was fed down the microcatheter, and the stent $(4 \times 20 \mathrm{~mm})$ was deployed through the thrombus by pulling back on the microcatheter. The device was withdrawn slowly with the microcatheter and thrombus and removed from the model MCA. Figure 5(b) shows a clot within the stent framework.

After each experiment, the catheters and devices were flushed with Hartmann's solution to remove any remaining lumen obstruction and air locks. Five attempts to retrieve the clot were allowed. If these were unsuccessful, the results were recorded as a fail. All experiments were performed by the same researcher.

2.4. Assessments. Recanalization was assessed visually and defined as complete removal of the thrombus with unobstructed free flow of Hartmann's solution through the previously occluded vessel. It was scored as 1 (successful complete removal) or 0 (unsuccessful or incomplete removal).

Clot fragmentation was assessed by inspection of the clot after retrieval and scored as 1 (more than one fragment) or 0 (no fragmentation/clot removed in one piece). 
TABLE 1: Summary of results for each device.

\begin{tabular}{|c|c|c|c|c|}
\hline Device & $4 \mathrm{~F}$ catheter & GPTAD & Solitaire & $P$-value \\
\hline Number of experiments $(n)$ & 26 & 30 & 26 & - \\
\hline Recanalization $(n,(\%))$ & $16(62 \%)$ & $23(77 \%)$ & $22(85 \%)$ & Ns* \\
\hline Fragmentation $(n,(\%))$ & $14(53 \%)$ & $7(23 \%)$ & $6(23 \%)$ & $<.05^{*}$ \\
\hline Number of attempts at retrieval (mean, (SD)) & $1.9(1.0)$ & $2.3(1.4)$ & $1.3(0.5)$ & $\mathrm{Ns} * *$ \\
\hline Time to successful recanalization (mean, SD) (s) & $41 \mathrm{SD} 42(n=16)$ & $61 \mathrm{SD} 21(n=23)$ & 197 SD $64(n=22)$ & $<.05^{* *}$ \\
\hline
\end{tabular}

${ }^{*}$ Chi-squared test $\left(2 \times 3\right.$ table), ${ }^{* *}$ Kruksal-Wallis test, $n$ : number, SD: standard deviation.

The time required for complete clot removal was recorded. This was measured using a stopwatch from deployment of each device into the system to complete removal of the thrombus from the model MCA. The duration of the experiment was measured from deployment of the device to successful removal or to the end of the fifth attempt of removal (if removal was unsuccessful). Interaction between the thrombus and the thrombectomy device was assessed by visual inspection.

2.5. Statistical Methods. Descriptive statistics and counts were performed using Microsoft Excel (Microsoft Office XP, Microsoft Corporation, US) and tests of significance (as detailed in the text) were carried out using Minitab 15 (Minitab Inc., USA, http://www.minitab.com/) statistical software package.

\section{Results}

Eighty-two experiments were performed in total, and the results of the experiments are summarised in Table 1.

3.1. Clot Length before Extraction. The length of the clots occluding the MCA was $16-68 \mathrm{~mm}$ for the $4 \mathrm{~F}, 21-55 \mathrm{~mm}$ for the GPTAD, and $20-45 \mathrm{~mm}$ for the Solitaire.

3.2. Recanalization. There was no significant difference in the rate of recanalization between the three devices $(16 / 26$ $(62 \%)$ for the $4 \mathrm{~F}, 23 / 30(77 \%)$ for the GPTAD, and $22 / 26$ $(85 \%)$ for the Solitaire, $P=.2,2 \times 32$-tailed Chi-squared test). Individual $2 \times 2$ comparisons (2-tailed Fisher's exact test) between devices also show no significant differences ( $4 \mathrm{~F}$ versus GPTAD $P=.3,4 \mathrm{~F}$ versus Solitaire $P=.1$, and GPTAD versus Solitaire $P=.5$ ).

3.3. Clot Fragmentation. There were significant differences in the incidence of clot fragmentation between the three devices with rates of $14 / 26(53 \%)$ for the $4 \mathrm{~F}, 7 / 30(23 \%)$ for the GPTAD, and 6/26 (23\%) for the Solitaire $(P=.02,2 \times 3$ 2 -tailed Chi-squared test). Individual comparisons (2-tailed Fisher's exact tests) between devices show significantly more fragmentation with the $4 \mathrm{~F}$ than with the GPTAD $(P=.04)$ and the Solitaire $(P=.03)$ but no difference between the GPTAD and the Solitaire $(P=1.0)$.

3.4. Time Taken for Successful Recanalization. The mean time taken for successful recanalization was 41 SD $42 \mathrm{~s}(n=16)$ for the 4F, $61 \mathrm{SD} 21 \mathrm{~s}(n=23)$ for the GPTAD, and 197 SD $64 \mathrm{~s}(n=22)$ for the Solitaire $(P<.05$, Kruksal-Wallis). Individual comparisons between the 3 devices showed that the mean time taken to remove the clot was significantly longer with the Solitaire than with two aspiration devices $(P<.05$ Kruksal-Wallis one-way analysis of variance). There was no significant difference in the time taken for successful clot removal between the $4 \mathrm{~F}$ and the GPTAD.

3.5. Device-Clot Interaction. Device-clot interaction was determined by visual inspection and by subjective assessment of the procedure by the researcher conducting the experiment. With the distal device (Solitaire), the passage of the microguidewire and microcatheter past the thrombus was simple. The Solitaire device deployed into the thrombus and grasped it well within the struts (Figure 5(b)). It was also able to catch a fragmented clot if it embolized distally by pushing the device further down the tubing. Withdrawal had to be controlled done very slowly and, otherwise the thrombus was lost. The aspiration devices stretched the thrombus during the aspiration process. The thrombus engaged in the tip of the catheter and was either pulled out whole or it broke up, leaving one or more fragments in the model circulation. The GPTAD appeared to grasp the clot effectively, and the removal was simple and smooth. However, if the clot fragmented, the device would sometimes be blocked and would have to be cleared. This was done by removing the device from the system and flushing it. The $4 \mathrm{~F}$ catheter was effective for thrombus removal but often caused clot fragmentation immediately on interacting with the thrombus. When it removed the thrombus, it was effective and smooth, but sometimes interaction with the thrombus was lost.

3.6. Complications. There were no complications with the Solitaire or 4F devices. The GPTAD had to be passed carefully so that the plastic sheath around the tip did not collapse, which did occur but could be corrected.

\section{Discussion}

The main findings of the study are that all three devices successfully removed the majority of clots from the model MCA. There was no significant difference in overall recanalization rates between the three devices. The $4 \mathrm{~F}$ catheter was most likely to fragment the clot. Time to complete clot removal 
was faster with aspiration devices (4F and GPTAD) than with the Solitaire.

4.1. Recanalization. A meta-analysis of 53 studies involving 2066 patients has shown that recanalization is strongly associated with improved functional outcomes and improved mortality [17]. Our data show that there was no significant difference in the effectiveness of the three tested devices in achieving recanalization in this model. Recanalization rates were $15 \%$ higher with the GPTAD than with the $4 \mathrm{~F}$ and a further $8 \%$ higher with the Solitaire than with the GPTAD. Such small differences would require a much larger number of experiments to identify any statistical significance. Successful thromboaspiration using catheters of varying calibre $(4-8 \mathrm{~F})$ has been reported in vivo $[4,18-22]$. In this study, clot aspiration via the GPTAD was nonsignificantly more effective than via the $4 \mathrm{~F}$ catheter alone. This may be due to the creation of the vortex at the tip of the GPTAD, allowing for more controlled clot capture. There was also a trend towards higher removal rates with the Solitaire than with either aspiration device. It has previously been shown in animal studies that recanalization is better using distal devices compared to proximal devices [6]. Even higher recanalization rates of $90-100 \%$ have been achieved with the Solitaire Device in combination with thrombolysis in human case series $[13,14]$.

4.2. Fragmentation. This study showed significantly more clot fragmentation with two aspiration devices than with the Solitaire. However, only clots removed via the aspiration/thrombectomy devices were counted. Assessment, therefore, included thrombi which were removed in several segments but had no potential for distal embolization because the segments were aspirated before the final occlusive fraction of the clot has been removed, thus not leading to distal embolization. The clinical relevance of the clot fragmentation described here is, therefore, unclear.

4.3. Time Taken for Successful Recanalization. The time taken for successful recanalization was significantly less with the aspiration devices than with the Solitaire. Distal devices are more complex mechanically than proximal devices and take more time to deploy [6], hence, the longer time for the Solitaire device compared to the GPTAD and $4 \mathrm{~F}$ catheter. Time to clot capture in this in vitro model is considerably faster than in vivo, since the vessel approach length is much shorter, more easily visible, and less tortuous compared to the human vascular system, and this would affect the level of time differences between devices in vivo. However, while our experiments cannot be directly extrapolated to the in vivo state, the differences are due to the technique of deployment and are likely to be observable in a greater or lesser extent in vivo. The relative importance would be expected to be less with a more complex and prolonged approach time to the site of the thrombus. However, the disadvantage of the longer recanalization time for the Solitaire in this model has to be balanced against less clot fragmentation, which would be relevant in vivo.
4.4. Clot-Device Interaction. In this study, the GPTAD appeared to grasp the clot better than the $4 \mathrm{~F}$ catheter, with more successful first-attempt removals, and this may have caused less fragmentation. However, once the clot was grasped, clot removal was smooth. Both devices appeared to stretch the clot on removal. This has been shown to reduce vasospasm on studies in animals [6]. The GPTAD creates a vortex with suction and does not touch the clot during removal [7], thus reducing the risk of fragmentation compared to the $4 \mathrm{~F}$ catheter. The Solitaire device uses an open-slit, closed cell design which interacts with the thrombus, giving an optimal radial force, increasing the chance of clot trapping [19]. In this study, it ensnared clots up to $45 \mathrm{~mm}$ of length within the struts of the device. If the clot was partially stretched, it would ensnare further down the device.

4.5. Experiment Limitations. This is an experimental study and, therefore, does not reflect the physiological environment in vivo with regard to forces involved between the thrombus, the vessel wall, and devices and also vessel damage, perforation, and vasospasm [23]. Clot adherence to vessel walls cannot be replicated [12], and the Hartmann's solution used has different characteristics from blood, which may thrombolyse or form additional thrombi during thrombectomy [24]. These factors may further affect recanalization. The model MCA was straight, and the navigation of the sharp bends in the carotid artery was not reflected in the silicone tubing. The device diameters differed between the suction catheters and the Solitaire, which may have affected results. The clots could also not be fully standardised, as some fragmented more than others. Furthermore, optimal placement of the device was ensured by direct visualization. This cannot be done clinically, which may have increased the recanalization rate for the devices.

\section{Conclusion}

In this experimental setup, thromboaspiration was as effective as thrombectomy but faster. These experiments have been performed in a model of the cerebral circulation and should be confirmed using in vivo studies. A combination of proximal and distal approaches might increase speed and recanalization rate.

\section{Disclosure}

The GP thrombus aspiration device was invented by G. Pearce and N. Perkinson. Wolverhampton University, the employer of G. Pearce, holds the patent for the device. KIMAL, Uxbridge, UK has produced the device for G. Pearce. $\mathrm{T}$. Jones has been conducting evaluations of the device in conjunction with Birmingham University funded by Kimal.

\section{Acknowledgments}

N. Perkinson (Waikato, New Zealand) was the coinventor of the GP thrombus aspiration device with G. Pearce. The 
authors are grateful to ev3 Inc. for the lending of a Solitaire $\mathrm{AB}$ Revascularization Device for use in these experiments. The authors would like to thank Dr. E. Wong and Dr. P. Horrocks of Keele University School of Life Sciences, for statistical advice.

\section{References}

[1] L. Thomassen and S. J. Bakke, "Endovascular reperfusion therapy in acute ischaemic stroke," Acta Neurologica Scandinavica, vol. 115, supplement 187, pp. 22-29, 2007.

[2] J. P. Broderick, "Endovascular therapy for acute ischemic stroke," Stroke, vol. 40, supplement 1, pp. S103-S106, 2009.

[3] W. Hacke, M. Kaste, E. Bluhmki et al., "Thrombolysis with alteplase 3 to 4.5 hours after acute ischemic stroke," New England Journal of Medicine, vol. 359, no. 13, pp. 1317-1329, 2008.

[4] G. M. Nesbit, G. Luh, R. Tien, and S. L. Barnwell, "New and future endovascular treatment strategies for acute ischemic stroke," Journal of Vascular and Interventional Radiology, vol. 15, no. 1, pp. S103-S110, 2004.

[5] C. D. Gandhi, L. D. Christiano, and C. J. Prestigiacomo, "Endovascular management of acute ischemic stroke," Neurosurgical Focus, vol. 26, no. 3, p. E2, 2009.

[6] J. Gralla, G. Schroth, L. Remonda, K. Nedeltchev, J. Slotboom, and C. Brekenfeld, "Mechanical thrombectomy for acute ischemic stroke: thrombus-device interaction, efficiency, and complications in vivo," Stroke, vol. 37, no. 12, pp. 3019-3024, 2006.

[7] C. Brekenfeld, G. Schroth, M. El-Koussy et al., "Mechanical thromboembolectomy for acute ischemic stroke: comparison of the catch thromboectomy device and the Merci Retriever in vivo," Stroke, vol. 39, no. 4, pp. 1213-1219, 2008.

[8] G. Pearce, J. H. Patrick, and N. D. Perkinson, "A new device for the treatment of thromboembolic strokes," Journal of Stroke and Cerebrovascular Diseases, vol. 16, no. 4, pp. 167-172, 2007.

[9] G. Pearce, N. Perkinson, J. Patrick, A. Crowe, and F. Jaegle, "Modelling and laboratory investigation into the efficacy of a new mechanical thrombectomy device," Journal of the World Universities, vol. 1, pp. 145-153, 2008.

[10] G. Pearce, N. Perkinson, R. Andrews, and F. Jaegle, "The design, optimisation, and testing of a new mechanical clot retrieval device for use in vascular surgery," International Journal of Engineering Simulation, vol. 9, pp. 10-26, 2008.

[11] G. Pearce, N. D. Perkinson, J. Wong et al., "The "GP" mechanical thrombectomy device," Journal of Stroke and Cerebrovascular Diseases, vol. 18, no. 4, pp. 288-293, 2009.

[12] G. Pearce, R. N. D. Perkinson, J. Wong et al., "In vitro testing of a new aspiration thrombus device," Journal of Stroke and Cerebrovascular Diseases, vol. 19, no. 2, pp. 121-129, 2010.

[13] S. Nayak, G. Ladurner, and M. Killer, "Treatment of acute middle cerebral artery occlusion with a Solitaire $\mathrm{AB}$ stent: preliminary experience," British Journal of Radiology, vol. 83, no. 996, pp. 1017-1022, 2010.

[14] C. Castaño, L. Dorado, C. Guerrero et al., "Mechanical thrombectomy with the solitaire ab device in large artery occlusions of the anterior circulation: a pilot study," Stroke, vol. 41, no. 8, pp. 1836-1840, 2010.

[15] T. Z. Jones, G. Pearce, N. Perkins, C. Roffe, and J. Wong, "An in-vitro investigation into the efficacy of the GP device compared to a control device," International Journal of Stroke, vol. 4, no. 2, p. 13, 2009.
[16] Ev3-Solitaire FR revascularization device in-service video CD-ROM operating guidelines.

[17] J. H. Rha and J. L. Saver, "The impact of recanalization on ischemic stroke outcome: a meta-analysis," Stroke, vol. 38, no. 3, pp. 967-973, 2007.

[18] H. L. Lutsep, W. M. Clark, G. M. Nesbit, T. A. Kuether, and S. L. Barnwell, "Intraarterial suction thrombectomy in acute stroke," American Journal of Neuroradiology, vol. 23, no. 5, pp. 783-786, 2002.

[19] R. Chapot, E. Houdart, A. Rogopoulos, C. Mounayer, J. P. Saint-Maurice, and J. J. Merland, "Thromboaspiration in the basilar artery: report of two cases," American Journal of Neuroradiology, vol. 23, no. 2, pp. 282-284, 2002.

[20] K. Nedeltchev, L. Remonda, D. D. Do et al., "Acute stenting and thromboaspiration in basilar artery occlusions due to embolism from the dominating vertebral artery," Neuroradiology, vol. 46, no. 8, pp. 686-691, 2004.

[21] G. F. Xu, C. S. Dae, G. C. Choong et al., "Aspiration thrombectomy of acute complete carotid bulb occlusion," Journal of Vascular and Interventional Radiology, vol. 16, no. 4, pp. 539-542, 2005.

[22] B. T. Jankowitz, A. Aleu, R. Lin et al., "Endovascular treatment of basilar artery occlusion by manual aspiration thrombectomy," Journal of NeuroInterventional Surgery, vol. 2, pp. 110$114,2010$.

[23] J. Gralla, G. Schroth, L. Remonda et al., "A dedicated animal model for mechanical thrombectomy in acute stroke," American Journal of Neuroradiology, vol. 27, no. 6, pp. 13571361, 2006.

[24] T. Liebig, J. Reinartz, R. Hannes, E. Miloslavski, and H. Henkes, "Comparative in vitro study of five mechanical embolectomy systems: effectiveness of clot removal and risk of distal embolization," Neuroradiology, vol. 50, no. 1, pp. 43-52, 2008 . 


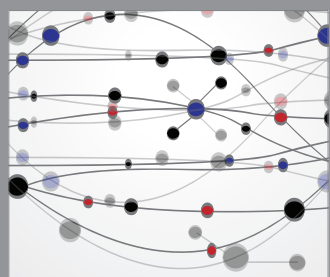

The Scientific World Journal
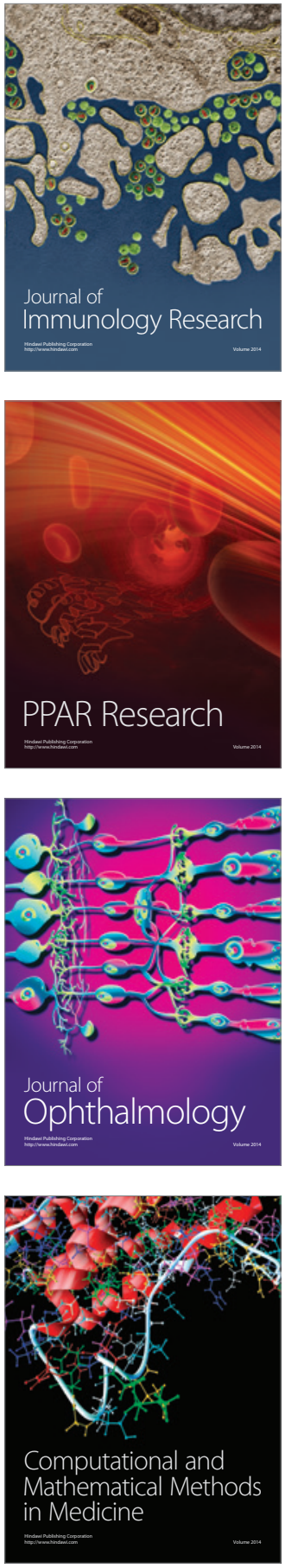

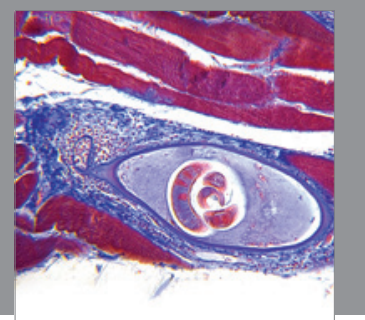

Gastroenterology

Research and Practice
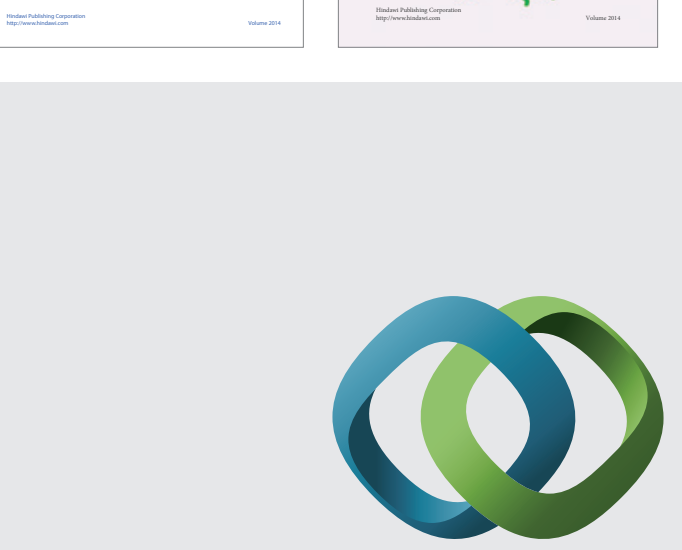

\section{Hindawi}

Submit your manuscripts at

http://www.hindawi.com
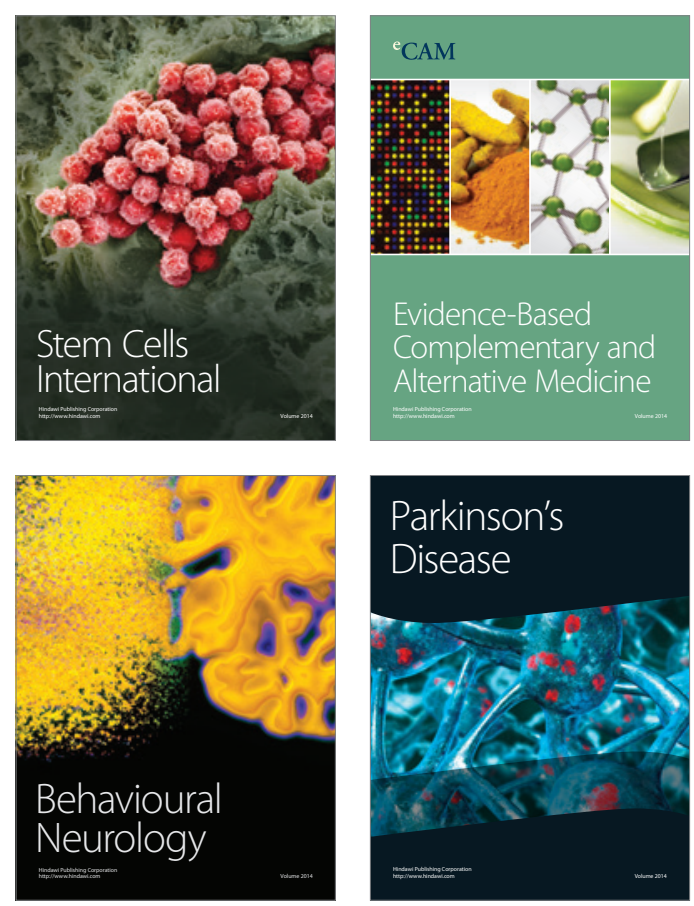

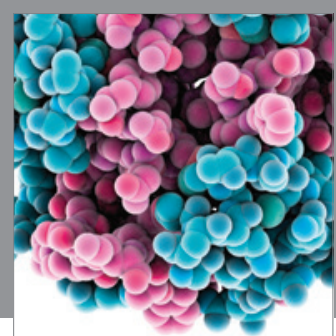

Journal of
Diabetes Research

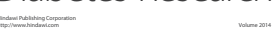

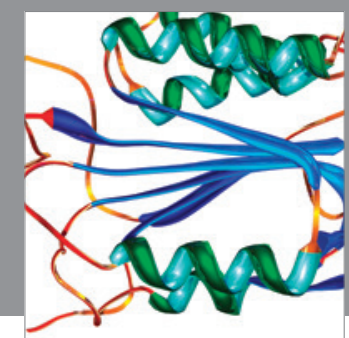

Disease Markers
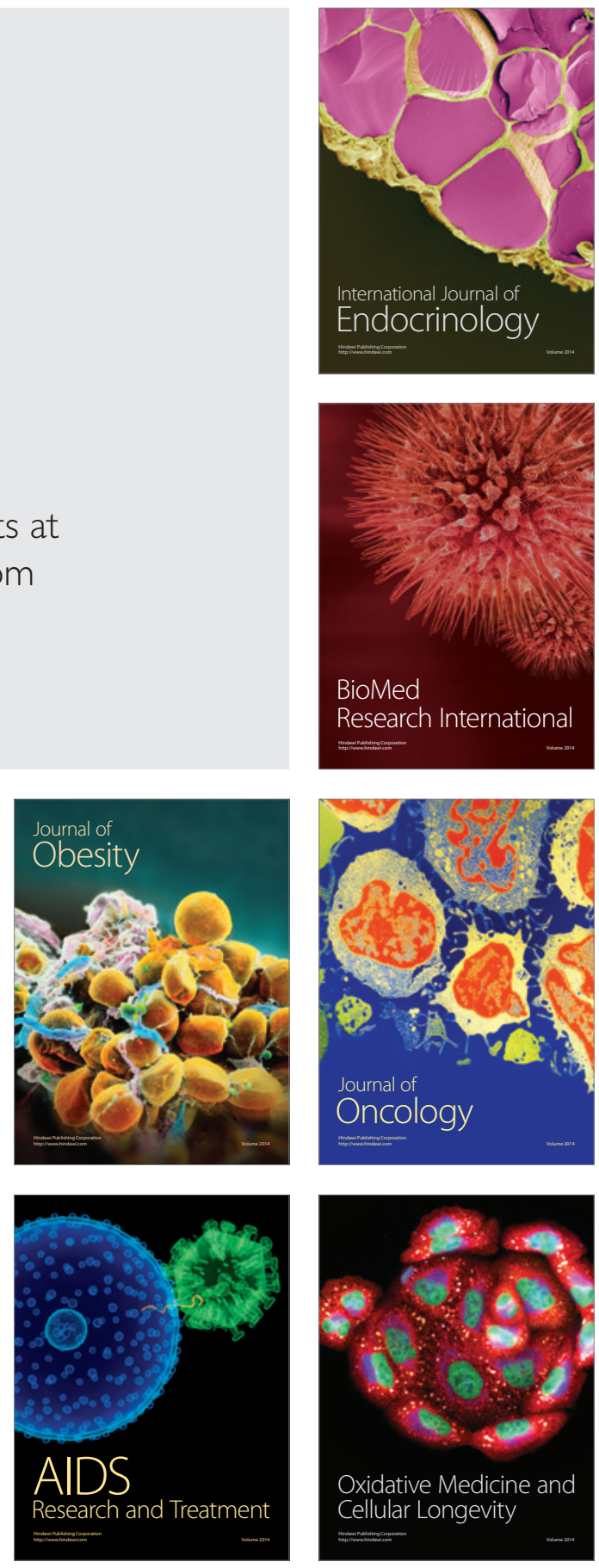